\title{
Health economics in mental health. 2: Economic evaluation
}

Neil Craig and Cameron Stark

This paper is the second in a series explaining key concepts and techniques used in health economics in the context of mental health care. The paper describes the different types of economic analysis and the circumstances in which they should be used. It explains key aspects of the methods used in economic evaluation to measure costs and benefits. The purpose of the paper is not to enable clinicians to undertake economic analysis, but to familiarise them with the methods used in economic evaluation and to enable them to assess the rigour and results of published studies.

Health care economic evaluation is a tool to inform decisions regarding the use of health care resources. There are several types of economic analysis. The method used must suit the decision being taken. Some of the concerns clinicians express regarding economic evaluation stem from the use of inappropriate analyses. Others are the result of a failure to distinguish between accountancy exercises, which simply attach a financial cost to a service, and the type of economic evaluation reviewed in this paper, which relate a full economic assessment of the opportunity costs of services to the benefits they generate.

This paper first describes how to estimate the cost of health services and then defines the different types of economic analysis and the circumstances in which they should be used. It describes and discusses the methods used to measure the benefits of health care in each type of evaluation, and briefly explains two key aspects of economic evaluation, discounting and sensitivity analysis.

\section{Costing methods}

Costing methods are common to all forms of economic evaluation. It is in costing methods that differences between economics and accountancy begin to emerge. Costs should be measured comprehensively (Knapp et al, 1990), and they should reflect the perspective from which the evaluation is being undertaken. For example, Knapp et al (1990) in their study of psychiatric reprovision for long-stay patients estimated the cost of accommodation and living expenses, hospital out-patient and day care, costs of health professionals including general practitioners (GPs), nursing and psychiatric services, nonhealth professional inputs, such as education, police and social work care, and other nonNational Health Service (NHS) costs such as voluntary services and patient travel costs. Knapp et al therefore took a societal perspective. If a narrow NHS perspective had been taken, then only costs falling on NHS organisations would have been measured. The risk in taking a narrower perspective is that apparent reductions (or increases) in NHS costs may disguise significant cost increases (or reductions) falling on other agencies, patients or carers.

Ideally, cost estimates should include the use of resources with no financial cost to the NHS, such as carer or voluntary sector input, or the value of land already in NHS possession, and they should include the opportunity cost of resources which have already been paid for such as existing buildings. They should also include the indirect and overhead cost of resources employed on a centralised basis within a hospital to support direct medical care such as administration and clinical support services. The principle is that any resource involved in the provision of a service which has an alternative use should be costed. That is, costs should reflect opportunity costs (Hallam et al, 1994).

Published studies do not always meet the standard. The economic argument surrounding the use of antipsychotic drugs such as clozapine or risperidone is sometimes discussed in terms of reduced hospital stays arising from use of the drugs (Addington et al, 1993; Reid et al, 1994). Yet the shorter stays need to be compared with increases in out-patient attendances, housing and primary care costs, and by additional costs to patients and carers arising from communitybased drug therapy (Meltzer et al, 1993).

Not all analyses need to include all relevant costs. The measurement of costs requires resources, and judgements need to be made as to whether the cost of increasing the scope and quality of cost information are justified by an 
improvement in accuracy which is likely to impact upon the results of the study. In the study by Knapp et al (1990), nursing services only accounted for $0.5 \%$ of the cost of community services for patients previously in long-term care, compared to $77.4 \%$ of costs accounted for by accommodation and living expenses. Clearly, it is more important to include and measure accurately the latter. Exclusion or approximation of the former would not materially affect the results. Whatever costs are included, the important point is that the costs considered and the assumptions used to estimate costs should be explicitly stated and they should reflect the perspective from which the evaluation is undertaken.

Costs of care depend on the treatment protocol. For example, Carpenter et al (1995) suggest that the cost of clozapine can be reduced by discontinuing treatment if benefit is not observed within 2-4 months. Relative costs of treatment also depend on the alternatives to the treatment under scrutiny. Addington et al (1993), for example, found that risperidone patients formed three subgroups: those who had never been hospitalised at all prior to prescription of risperidone; those who had been in hospital continuously in the year prior to starting treatment; and those who had spent time in hospital in the preceding year. The cost and the treatment outcomes of the treatment pre-risperidone may differ for the three groups, and therefore the relative cost-effectiveness of risperidone therapy will vary according to which of the three groups patients would belong to in the absence of the therapy. Treatment protocols and comparison groups should therefore be clearly explained to enable judgements to be made as to the validity and generalisation of results.

The way costs are allocated can be of particular importance in costing pilot projects, as they often have non-recurring start-up costs, together with start-up and winding down periods in which throughput or case-load will be lower than normal. Comparisons of cost per case and other measures of cost-effectiveness should be made based on levels of activity expected once the service is fully operational (Hallam et al, 1994), although non-recurring costs and double-running costs of keeping existing facilities and services open while new services are developed have to be considered when planning the implementation of the results of pilot projects (Knapp et al, 1990; Kavanagh, 1993; Rosenheck et al, 1995).

\section{Types of economic evaluation}

The nature and measurement of outcomes defines the particular kind of evaluation chosen. a choice which should be made in light of the study question being addressed.

\section{Cost minimisation analysis}

CMA is used where there is evidence that the outcomes of two interventions are the same. If it has been found, for example, that $50 \%$ of patients with depression in receipt of either drug or cognitive therapy have fully recovered by six months, then the treatments can be considered to be equally effective, and the economic evaluation can concentrate on costs. The treatment of choice on efficiency grounds would be the least costly service.

Burns et al (1993) examined the treatment patterns and costs of an experimental homebased acute psychiatric service compared to standard psychiatric care. Clinical and social outcomes were similar in control and experimental groups, yet costs were significantly lower for the home-based service, largely due to reduced frequency and duration of hospital admissions. Because the services achieved similar outcomes, the judgement on the relative efficiency of the two services could be based on comparative costs alone, the authors concluding that the home-based service was more costeffective.

\section{Cost-effectiveness analysis}

A more common scenario is that treatments will differ in terms of both costs and effectiveness. If for every 100 affected patients, therapy ' $x$ ' cures 50 patients at a cost of $£ 100000$ and therapy ' $y$ ' cures 25 patients at a cost of $£ 75000$, therapy ' $x$ ' would be the more cost-effective of the two treatments at a cost of $\$ 2000$ per cured patient as opposed to $£ 3000$ per cured patient for therapy ' $y$ '.

Cost effectiveness analysis (CEA) addresses issues of technical efficiency. The objective or outcome measure, in this example, a cured patient, is not questioned. CEA establishes the intervention which has the maximum impact on the objective in question in relation to the costs of the different interventions.

A limitation of CEA is that in general most interventions have more than one objective and impact upon more than one outcome variable. Drug therapies may have physiological sideeffects. Clozapine, for example, can cause agranulocytosis which in rare cases is fatal and which has knock-on consequences for the cost of treatment in terms of blood monitoring to avert this risk. Outcomes usually have several dimensions which, especially in mental health. may be hard to quantify making costs per unit of outcome difficult to define. Useful analyses can still be undertaken (Goldberg, 1995). Knapp et 
al $(1994 a, b)$, for example, compared the costeffectiveness of in-patient services within various forms of community mental health services. They found community options in general to be more, or at least as, effective on a range of measures of psychosocial well-being, while being less expensive. Without having to express overall cost-effectiveness as a single measure, they concluded that community care was more cost-effective.

\section{Cost utility analysis}

CUA can be used where treatment affects several dimensions of health. Longevity resulting from different treatments is adjusted to reflect the quality of life enjoyed over patients' remaining years of life. Treatment outcomes are expressed in terms of quality adjusted life years (QALYs).

Several ethical and practical issues are raised by the use of GALYs (Mooney, 1989; Rawles, 1989; Carr-Hill \& Morris, 1991). There are, for example, concerns regarding the dimensions of health included within measures of quality of life. The measure which has been most commonly used in economic studies to date has been the Rosser-Watts-Kind matrix which categorises health states in terms of two dimensions, pain and disability.

This measure along with many other quality of life measures, is insensitive to dimensions of health which are important in chronic and/or psychosocial conditions affecting activities of daily living. Mental health includes other important dimensions such as stress, anxiety or depression (Donaldson et al, 1988; Wilkinson et al, 1992). The impact of using measures which exclude such variables would be to bias assessments of cost-effectiveness.

Measures are required which express multidimensional quality of life in a single, quantitative measure. Validated, reliable measures are available which address single important dimensions of mental health, but combining them into a single, quantitative measure which can be used to compare the impact on health of different treatments affecting different dimensions of health presents unresolved methodological problems. For example, evidence suggests that people currently experiencing ill-health value health states differently to healthy respondents (Kind \& Dolan, 1995).

Attempts have been made to derive single multidimensional measures of health (EurogoL Group, 1990). For example, in the EurogoL, respondents value, in relation to perfect health. health states comprising pre-defined levels of several dimensions of health, including physical, mental and activities of daily living variables. However, this work is still in the developmental stage and few cost utility ana- lyses, in particular in the field of mental health, have been undertaken which use multidimensional quality of life measures.

\section{Cost-benefit analysis}

Like CUA, CBA is a technique for evaluating interventions which have multiple outcomes. It differs from CUA in three main respects. First. in addition to health benefits, it evaluates nonhealth benefits arising from a particular intervention, such as improvements in patient access, reduced waiting times or the productive gains from reduced time off work. Second, in order to make these benefits comparable both with each other and with the costs of the intervention, benefits are expressed in money terms. Third, CBA does not address the relative effectiveness of ways of meeting a given objective, such as GALYs or, in the context of CEA, objectives such as episodes of depression prevented. Rather, total benefits are compared with total costs to assess whether the treatment is worthwhile in economic terms, that is, whether benefits exceed costs. If two treatments are being compared, the treatment with the greater excess of benefit over cost would be the preferred treatment.

Non-health benefits can be measured in a number of ways. Analyses which include the productive gains resulting from effective treatments which reduce time off work infer the value of such benefits from the earnings of the patient or patient groups receiving the treatment. This raises the ethical issue of potential bias against groups who are not economically active, such as the elderly or the unemployed. Another nonhealth benefit is improvement in patient access which can be measured in terms of the reduced cost of travel to the health care setting in question and reduced travel time, again valued in terms of hourly earnings.

Valuing health benefits in monetary terms is more problematic. The methods used to value health directly in financial terms fall into two main categories. The first involves inferences of the value people attach to their lives or good health from the financial decisions they take, or which government or other agencies take on their behalf, which impact upon mortality or morbidity. This method is called implicit valuation. The second method, referred to as contingent valuation, asks representative samples of the public. patients or carers how much they would be willing to pay to reduce the risk of death or illness. The value of treatments which reduce the risk of death or illness is then inferred from the responses.

An example of the kind of information used in implicit valuation is the danger money paid to people in high risk employment. Such risk 
premiums imply a value attached to reduced risk of death or illness. Several problems arise in inferring these values. First, a range of factors over and above people's attitude to risk and the value they attach to good health determines the decisions people take. Second, the assumption that people know and understand the risks they face is untenable. Third, individuals' (and governments') ability to pay is not necessarily the same as their willingness to pay. The decisions they take are inevitably constrained by their income.

Similarly, responses derived using contingent valuation may be affected by income. Questions need to be framed carefully to make responses valid and reliable. Progress has been made in developing these techniques but debate continues within and outwith the economics field over the extent to which they provide valid and reliable information for use in economic evaluation.

\section{Discounting}

Discounting is the technique used by economists to take account of the phasing of costs and benefits. Most people given the choice of making payments now or in the future would choose the latter, whereas given the option of receiving income or enjoying benefits now or in the future they would choose the former. Economists refer to this as time preference. The real value of a given cost or benefit decreases the farther into the future the cost is incurred or the benefit is received. The rate at which the value decreases is called the 'discount rate'. It is conventional to use a discount rate of $6 \%$ per year.

Higher discount rates favour treatments with moderate initial costs and immediate benefits, such as drug treatments, over treatments involving substantial initial investment for long-term gains, such as cognitive therapy for depression, which has higher initial costs but may reduce relapse in the future. The discount rate embodies value judgements about how much society as a whole is prepared to defer costs and benefits. Economic evaluations should test the sensitivity of results to different value judgements using a range of discount rates.

\section{Sensitivity analysis}

The discussion of costing shows that comprehensive costings require a lot of information, which might not always be available and which is time-consuming and costly to gather. Most evaluations will involve compromises between the ideal of accurate, comprehensive costings and what is feasible with the resources, time and information available. The discussion of outcome measurement illustrates that the methods available vary in terms of sensitivity, validity, reliability and comparability. A degree of uncertainty therefore surrounds both costs and outcomes in economic evaluation. The picture is complicated further by the unpredictable nature of both costs and outcomes in health care.

Economic evaluation should always include analysis of the sensitivity of conclusions to these uncertainties and risks. Where there is doubt surrounding cost or outcome information used in an evaluation, alternative assumptions regarding this information should be made and the results reworked to see whether they affect the original conclusions. For example. Burns et al (1993) tested the sensitivity of their conclusions regarding the efficiency of an experimental home-based acute psychiatric service to increases in the estimated cost of home visits. The relative cost-effectiveness of the service was unaffected by the results. Likewise, Davies $\&$ Drummond (1993) tested the sensitivity of their results regarding the cost-effectiveness of clozapine to the assumptions they had made in their analysis. Again, the results were unaffected.

\section{Conclusion}

Financial stringency in health care is familiar to all. Even if health service commissioners have access to increased resources in the future, health economics is likely to be one of the main methods used to assist decisions on resource use. Mental health services will find themselves the subject of economic evaluation, and will often find that development bids are subject to economic scrutiny. It is important, therefore that clinicians are able to interpret and evaluate the results of economic evaluations.

\section{References}

AdDington, D. E., Jones, B., BLOOM, D., et al (1993) Reduction of hospital days in chronic schizophrenic patients treated with risperidone: A retrospective study. Clinical Therapeutics, 16, 917-926.

BURNS, T.. RAFTERY, J., BEADSMOORE. A.. et al (1993) A controlled trial of home-based acute psychiatric services. II: Treatment patterns and costs. British Journal of Psychiatry. 163, 55-61.

Carpenter, W. T., Conley, R. R. Buchanan, R. W., et al (1995) Patient response and resource management. Another view of clozapine treatment of schizophrenia. American Joumal of Psychiatry. 162. 827-832.

CARR-HILL. R. \& MORRIS, J. (1991) Current practice in obtaining the ' $Q$ ' in GALYs: a cautionary note. British Medical Journal, s03, 699-701.

DAVIES, L. M. \& DRUMMOND. M. F. (1993) Assessment of costs and benefits of drug therapy for treatmentresistant schizophrenia in the UK. British Journal of Psychiatry. 162. 38-42. 
Donaldson, C.. ATKInson, K., Bond, J., et al (1988) Should QALYs be programme-specific? Journal of Health Economics, 7, 239-257.

EUROQOL GROUP (1990) Eurogol: a new facility for the measurement of health-related quality of life. Health Policy. 16, 199-208.

GOLDBERG, D. (1995) Cost-effectiveness studies in the valuation of mental health services in the community: current knowledge and problems. International Clinical Psychopharmacology, 8 (suppl. 5), 29-34.

Hallam, A., BEecham, J., KNAPP, M.. et al (1994) The costs of accommodation and care: Community provision for former long-stay psychiatric hospital patients. European Archives of Psychiatry and Clinical Neurosctences, 243, 304-310.

KaVANAGH, S. (1993) Elderly people with cognitive impairment: Costing possible changes in the balance of care. Health and Social Care in the Community, 1, 69-80.

KIND. P. \& DoLAN. P. (1995) The effect of past and present illness experience on the valuation of health states. Medical Care, 33, AS255-AS263.

KNAPP, M. BEECHAM, J., ANDERSON, J., et al (1990) The TAPS Project. 3: Predicting the community costs of closing psychiatric hospitals. British Journal of Psychiatry. 157, 661-670.

- - Koutsogeorgopoulou, V., et al (1994a) Service use and costs of home-based versus hospital-based care for people with serious mental illness. British Journal of Psychiatry. 165, 195-203.

-. CAmbridge, P.. Thomason, C., et al (1994b) Residential care as an alternattve to long-stay hospital: a costeffectiveness evaluation of two pilot projects. International Joumal of Geriatric Psychiatry, 9. 297-304.
MELTZER, H. Y. COLA, P., WAY, L., et al (1993) Costeffectiveness of clozapine in neuroleptic-resistant schizophrenia. American Joumal of Psychiatry, 160. 1630-1638.

MOONEY, G. (1989) GALYs: Are they enough? A health economist's perspective. Journal of Medical Ethics. 18. 148-152.

RAwLES. J. M. (1989) Castigating QALYs. Joumal of Medical Ethics. 15. 143-147.

REID. W. H., MASON, M. \& TOPRAC. M. (1994) Savings in hospital bed-days related to treatment with clozapine. Hospital and Community Psychiatry, 46. 261-263.

Rosenheck, R., Neale, M. \& Frisman, L. (1995) lssues in estimating the cost of innovative mental health programs. Psychiatric Quarterly, 66, 9-31.

WILKINSON, G.. WILLAMS, B., KREKORIAN, H., et al (1992) QALYs in mental health: a case study. Psychological Medicine, 22, 725-731.

*Neil Craig, Health Economist, Department of Public Health, University of Glasgow, 2 Lilybank Gardens, Glasgow G12 8RZ; and Cameron Stark, Consultant in Public Health Medicine, Highland Health Board, Beechwood Park, Inverness IV2 3HG

*Correspondence 